\title{
Algebras and modules in monoidal model categories
}

\author{
Stefan Schwede And Brooke E. Shipley ${ }^{1}$
}

\begin{abstract}
We construct model category structures for monoids and modules in symmetric monoidal model categories, with applications to symmetric spectra and $\Gamma$-spaces.
\end{abstract}

1991 AMS Math. Subj. Class.: primary 55U35, secondary 18D10

\section{Summary}

This paper gives a general approach for obtaining model category structures for algebras or modules over some other model category. Technically, what we mean by an 'algebra' is a monoid in a symmetric monoidal category. Of course, the symmetric monoidal and model category structures have to be compatible, which leads to the definition of a monoidal model category, see Definition 2.1. To obtain a model category structure of algebras we have to introduce one further axiom, the monoid axiom (Definition 2.2). A filtration on certain pushouts of monoids (see Lemma 5.2) is then used to reduce the problem to standard model category arguments based on Quillen's "small object argument". Our main result is stated in Theorem 3.1.

This approach was developed in particular to apply to the category of symmetric spectra defined in [HSS] and to $\Gamma$-spaces in [Sch2]. In both of these categories we thus obtain model categories for the associative monoids, the $R$-modules for any monoid $R$, and the $R$-algebras for any commutative monoid $R$. A significant shortcut is possible if the underlying monoidal model category has the special property that all objects are fibrant, see Remark 3.5. This is not true for our main examples, symmetric spectra and $\Gamma$-spaces. It does hold though, in the monoidal model categories of simplicial abelian groups, chain complexes, or $S$-modules (in the sense of $[\mathrm{EKMM}])$.

We assume that the reader is familiar with the language of homotopical algebra (cf. [Q], [DS]) and with the basic ideas concerning monoidal and symmetric monoidal categories (cf. [MacL, VII], [Bor, 6]) and triples (also called monads, cf. [MacL, VI.1], [Bor, 4]).

Acknowledgments. We would first like to thank Charles Rezk for conversations which led us to the filtration that appears in Lemma 5.2. We also benefited from several conversations about this project with Bill Dwyer, Mark Hovey and Manos Lydakis. We would also like to thank Bill Dwyer, Phil Hirschhorn, and Dan Kan for sharing the draft of [DHK] with us. In Appendix A we recall the notion of a cofibrantly generated model category from their book.

\footnotetext{
${ }^{1}$ Research partially supported by an NSF Postdoctoral Fellowship
} 


\section{Monoidal model categories}

A monoidal model category is essentially a model category with a compatible closed symmetric monoidal product. The compatibility is expressed by the pushout product axiom below. In this paper we always require a closed symmetric monoidal product although for expository ease we refer to these categories as just 'monoidal' model categories. One could also consider model categories enriched over a monoidal model category with certain compatibility requirements analogous to the pushout product axiom or the simplicial axiom of [Q, II.2]. For example, closed simplicial model categories [Q, II.2] are such compatibly enriched categories over the monoidal model category of simplicial sets.

We also introduce the monoid axiom which is the crucial ingredient for lifting the model category structure to monoids and modules. Examples of monoidal model categories satisfying the monoid axiom are given in Section 4.

Definition 2.1 A model category $\mathcal{C}$ is a monoidal model category if it is endowed with a closed symmetric monoidal structure and satisfies the following pushout product axiom. We will denote the symmetric monoidal product by $\wedge$, the unit by $\mathbb{I}$ and the internal Hom object by $[-,-]$.

Pushout product axiom. Let $A \longrightarrow B$ and $K \longrightarrow L$ be cofibrations in $\mathcal{C}$. Then the map

$$
A \wedge L \cup_{A \wedge K} B \wedge K \longrightarrow B \wedge L
$$

is also a cofibration. If in addition one of the former maps is a weak equivalence, so is the latter map.

If $\mathcal{C}$ is a category with a monoidal product $\wedge$ and $I$ is a class of maps in $\mathcal{C}$, we denote by $I \wedge \mathcal{C}$ the class of maps of the form

$$
A \wedge Z \longrightarrow B \wedge Z
$$

for $A \longrightarrow B$ a map in $I$ and $Z$ an object of $\mathcal{C}$. We also denote by $I$-cof feg $_{\text {reg }}$ the class of maps obtained from the maps of $I$ by cobase change and composition (possibly transfinite, see Appendix A.) These maps are referred to as the regular I-cofibrations.

Definition 2.2 A monoidal model category $\mathcal{C}$ satisfies the monoid axiom if every map in

$$
(\{\text { acyc. cofibrations }\} \wedge \mathcal{C})-\text { cof } f_{\text {reg }}
$$

is a weak equivalence.

Note that if $\mathcal{C}$ has the special property that every object is cofibrant, then the monoid axiom is a consequence of the pushout product axiom. However, this special situation rarely occurs in practice.

In Appendix A we recall cofibrantly generated model categories. In these model categories fibrations can be detected by checking the right lifting property against a set of maps, called generating acyclic cofibrations, and similarly for acyclic fibrations. This is in contrast to general model categories where the lifting property has to be checked against the whole class of acyclic cofibrations. In cofibrantly generated model categories, the pushout product axiom and the monoid axiom only have to be checked for the generating (acyclic) cofibrations: 
Lemma 2.3 Let $\mathcal{C}$ be a cofibrantly generated model category endowed with a closed symmetric monoidal structure.

1. If the pushout product axiom holds for the generating cofibrations and the generating acyclic cofibrations, then it holds in general.

2. Let $J$ be a set of generating acyclic cofibrations. If every map in $(J \wedge \mathcal{C})$-cof reg is a weak equivalence, then the monoid axiom holds.

Proof: For the first statement consider a map $i: A \longrightarrow B$ in $\mathcal{C}$. Denote by $G(i)$ the class of maps $j: K \longrightarrow L$ such that the pushout product

$$
A \wedge L \cup_{A \wedge K} B \wedge K \longrightarrow B \wedge L
$$

is a cofibration. This pushout product has the left lifting property with respect to a map $f: X \longrightarrow Y$ if and only if $j$ has the left lifting property with respect to the map

$$
p:[B, X] \longrightarrow[B, Y] \times_{[A, Y]}[A, X] .
$$

Hence, a map is in $G(i)$ if and only if it has the left lifting property with respect to the map $p$ for all $f: X \longrightarrow Y$ which are acyclic fibrations in $\mathcal{C}$.

$G(i)$ is thus closed under cobase change, transfinite composition and retracts. If $i: A \longrightarrow B$ is a generating cofibration, $G(i)$ contains all generating cofibrations by assumption; because of the closure properties it thus contains all cofibrations, see Lemma A.1. Reversing the roles of $i$ and an arbitrary cofibration $j: K \longrightarrow L$ we thus know that $G(j)$ contains all generating cofibrations. Again by the closure properties, $G(j)$ contains all cofibrations, which proves the pushout product axiom for two cofibrations. The proof of the pushout product being an acyclic cofibration when one of the constituents is, follows in the same manner.

For the second statement note that by the small object argument, Lemma A.1, every acyclic cofibration is a retract of a transfinite composition of cobase changes along the generating acyclic cofibrations. Since transfinite compositions of transfinite compositions are transfinite compositions, every map in $(\{$ acyc. cofibrations $\} \wedge \mathcal{C})$-cof reg is thus a retract of a map in $(J \wedge \mathcal{C})$ cof $_{\text {reg }}$.

\section{$3 \quad$ Model categories of algebras and modules}

In this section we state the main theorem, Theorem 3.1, which constructs model categories for algebras and modules. The proof of this theorem is delayed to section 5. Examples of model categories for which this theorem applies are given in section 4 . We end this section with two theorems which compare the homotopy categories of modules or algebras over weakly equivalent monoids.

We consider a symmetric monoidal category with product $\wedge$ and unit II. A monoid is an object $R$ together with a "multiplication" map $R \wedge R \longrightarrow R$ and a "unit" $\mathbb{I} \longrightarrow R$ which satisfy certain associativity and unit conditions (see [MacL, VII.3]). $R$ is a commutative monoid if the multiplication map is unchanged when composed with the twist, or the symmetry isomorphism, of $R \wedge R$. If $R$ is a monoid, a left $R$-module ("object with left $R$-action" in [MacL, VII.4]) is an 
object $N$ together with an action map $R \wedge N \longrightarrow N$ satisfying associativity and unit conditions (see again [MacL, VII.4]). Right $R$-modules are defined similarly.

Assume that $\mathcal{C}$ has coequalizers. Then there is a smash product over $R$, denoted $M \wedge{ }_{R} N$, of a right $R$-module $M$ and a left $R$-module $N$. It is defined as the coequalizer, in $\mathcal{C}$, of the two maps $M \wedge R \wedge N \Longrightarrow M \wedge N$ induced by the actions of $R$ on $M$ and $N$ respectively. If $R$ is a commutative monoid, then the category of left $R$-modules is isomorphic to the category of right $R$-modules, and we simply speak of $R$-modules. In this case, the smash product of two $R$-modules is another $R$-module and smashing over $R$ makes $R$-mod into a symmetric monoidal category with unit $R$. If $\mathcal{C}$ has equalizers, there is also an internal Hom object of $R$-modules, $[M, N]_{R}$. It is the equalizer of two maps $[M, N] \Longrightarrow[R \wedge M, N]$. The first map is induced by the action of $R$ on $M$, the second map is the composition of $R \wedge-:[M, N] \longrightarrow[R \wedge M, R \wedge N]$ followed by the map induced by the action of $R$ on $N$.

For a commutative monoid $R$, an $R$-algebra is defined to be a monoid in the category of $R$ modules. It is a formal property of symmetric monoidal categories (cf. [EKMM, VII 1.3]) that specifying an $R$-algebra structure on an object $A$ is the same as giving $A$ a monoid structure together with a monoid map $f: R \longrightarrow A$ which is central in the sense that the following diagram commutes.

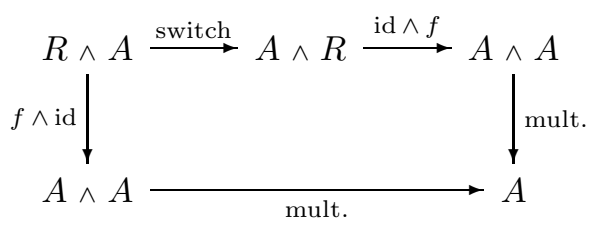

Now we can state our main theorem. It essentially says that monoids, modules and algebras in a cofibrantly generated, monoidal model category $\mathcal{C}$ again form a model category if the monoid axiom holds. (See Appendix A for the definition of a cofibrantly generated model category.) To simplify the exposition, we assume that all objects in $\mathcal{C}$ are small (refer to Appendix A) relative to the whole category. This last assumption can be weakened as indicated in A.5. The proofs will be delayed until the last section.

In the categories of monoids, left $R$-modules (when $R$ is a fixed monoid), and $R$-algebras (when $R$ is a commutative monoid) a morphism is defined to be a fibration or weak equivalence if it is a fibration or weak equivalence in the underlying category $\mathcal{C}$. A morphism is a cofibration if it has the left lifting property with respect to all acyclic fibrations.

Theorem 3.1 Let $\mathcal{C}$ be a cofibrantly generated, monoidal model category. Assume further that every object in $\mathcal{C}$ is small relative to the whole category and that $\mathcal{C}$ satisfies the monoid axiom.

1. Let $R$ be a monoid in $\mathcal{C}$. Then the category of left $R$-modules is a cofibrantly generated model category.

2. Let $R$ be a commutative monoid in $\mathcal{C}$. Then the category of $R$-modules is a cofibrantly generated, monoidal model category satisfying the monoid axiom.

3. Let $R$ be a commutative monoid in $\mathcal{C}$. Then the category of $R$-algebras is a cofibrantly generated model category. If the unit $\mathbb{I}$ of the smash product is cofibrant in $\mathcal{C}$, then every cofibration of $R$-algebras whose source is cofibrant in $\mathcal{C}$ is also a cofibration of $R$-modules. In particular, any cofibrant $R$-algebra is cofibrant as an $R$-module.

If in part (3) of the theorem we take $R$ to be the unit of the smash product, we see that in particular the category of monoids in $\mathcal{C}$ forms a model category. 
Remark 3.2 The full strength of the monoid axiom is not necessary to obtain a model category of $R$-modules for a particular monoid $R$. In fact, to get hypothesis (1) of Lemma A.3 for $R$ modules, one need only know that every map in

$$
(\{\text { acyc. cofibrations }\} \wedge R)-\text { cof }_{\text {reg }}
$$

is a weak equivalence. This holds, independent of the monoid axiom, if $R$ is cofibrant in the underlying category $\mathcal{C}$. For then the pushout product axiom implies that smashing with $R$ preserves acyclic cofibrations.

The following theorems concern comparisons of homotopy categories of modules and algebras. The homotopy theory of $R$-modules and $R$-algebras should only depend on the weak equivalence type of the monoid $R$. To show this for $R$-modules we must require that the functor $-\wedge{ }_{R} N$ take any weak equivalence of right $R$-modules to a weak equivalence in $\mathcal{C}$ whenever $N$ is a cofibrant left $R$-module. In all of our examples this added property of the smash product holds. For the comparison of $R$-algebras, we also require that the unit of the smash product is cofibrant.

Theorem 3.3 Assume that for any cofibrant left $R$-module $N,-\wedge_{R} N$ takes weak equivalences of right $R$-modules to weak equivalences in $\mathcal{C}$. If $R \stackrel{\sim}{\longrightarrow} S$ is a weak equivalence of monoids, then the total derived functors of restriction and extension of scalars induce equivalences of homotopy categories

$$
\mathrm{Ho}(R \text {-mod }) \cong \mathrm{Ho}(S \text {-mod }) \text {. }
$$

Proof: This is an application of Quillen's adjoint functor theorem ([Q, I.4 Thm. 3] or [DS, Thm. 9.7]). The weak equivalences and fibrations are defined in the underlying symmetric monoidal category, hence the restriction functor preserves fibrations and acyclic fibrations. By assumption, for $N$ a cofibrant left $R$-module

$$
N \cong R \wedge{ }_{R} N \longrightarrow S \wedge{ }_{R} N
$$

is a weak equivalence. Thus if $Y$ is a fibrant left $S$-module, an $R$-module map $N \longrightarrow Y$ is a weak equivalence if and only if the adjoint $S$-module map $S \wedge R N \longrightarrow Y$ is a weak equivalence. [DS, Thm. 9.7] then gives the desired result.

Theorem 3.4 Suppose that the unit $\mathbb{I}$ of the smash product is cofibrant in $\mathcal{C}$ and that for any cofibrant left $R$-module $N,-\wedge_{R} N$ takes weak equivalences of right $R$-modules to weak equivalences in $\mathcal{C}$. Then for a weak equivalence of commutative monoids $R \stackrel{\sim}{\longrightarrow} S$, the total derived functors of restriction and extension of scalars induce equivalences of homotopy categories

$$
\mathrm{Ho}(R \text {-alg }) \cong \mathrm{Ho}(S \text {-alg }) .
$$

Proof: The proof is similar to the one of the previous theorem. Again the right adjoint restriction functor does not change underlying objects, so it preserves fibrations and acyclic fibrations. Since cofibrant $R$-algebras are also cofibrant as $R$-modules (Thm. $3.1(3)$ ), for any cofibrant $R$-algebra the adjunction morphism is again a weak equivalence. So [DS, Thm. 9.7] applies one more time.

Remark 3.5 Some important examples of monoidal model categories have the property that all objects are fibrant. This greatly simplifies the situation. If there is also a simplicial or topological model category structure and if a simplicial (resp. topological) triple $T$ acts, then the category of $T$-algebras is again a simplicial (topological) category, so it has path objects. Hence hypothesis (2) of Lemma A.3 applies. One example of this situation is the category of $S$-modules in [EKMM]. Lemma A.3 (2) should be compared to [EKMM, Thm. VII 4.7]. 
Remark 3.6 We point out again that in our main examples, symmetric spectra and $\Gamma$-spaces, not all objects are fibrant, which is why we need a more complicated approach. In the fibrant case, one gets model category structures for algebras over all reasonable (e.g. continuous or simplicial) triples, whereas our monoid axiom approach only applies to the free $R$-module and free $R$-algebra triples. The category of commutative monoids often has a model category structure in the fibrant case (e.g. commutative simplicial rings or commutative $S$-algebras [EKMM, Cor. VII 4.8]). In contrast, for $\Gamma$-spaces and symmetric spectra, the category of commutative monoids can not form a model category with fibrations and weak equivalences defined in the underlying category. For if such a model category structure existed, one could choose a fibrant replacement of the unit $S^{0}$ inside the respective category of commutative monoids. Evaluating this fibrant representative on $1^{+} \in \Gamma^{\mathrm{op}}$, or at level 0 respectively, would give a commutative simplicial monoid weakly equivalent to $Q S^{0}$. This would imply that the space $Q S^{0}$ is weakly equivalent to a product of Eilenberg-MacLane spaces, which is not the case. The homotopy category of commutative monoids in symmetric spectra is still closely related to $E_{\infty}$-ring spectra, though.

\section{Examples}

\section{Simplicial sets.}

The category of simplicial sets has a well-known model category structure established by D. Quillen [Q, II 3, Thm. 3]. The cofibrations are the degreewise injective maps, the fibrations are the Kan fibrations and the weak equivalences are the maps which become homotopy equivalences after geometric realization. This model category is cofibrantly generated. The standard choice for the generating (acyclic) cofibrations are the inclusions of the boundaries (resp. horns) into the standard simplices. Here every object is small with respect to the whole category.

The cartesian product of simplicial sets is symmetric monoidal with unit the discrete one-point simplicial set. The pushout product axiom is well-known in this case, (see [GZ, IV Prop. 2.2], [Q, II 3, Thm. 3]). Since every simplicial set is cofibrant, the monoid axiom follows from the pushout product axiom. A monoid in the category of simplicial sets under cartesian product is just a simplicial monoid, i.e., a simplicial object of ordinary unital and associative monoids. So the main theorem, Theorem 3.1 (3), recovers Quillen's model category structure for simplicial monoids [Q, II 4, Thm. 4, and Rem. 1, p. 4.2].

\section{$\Gamma$-spaces and symmetric spectra}

These two examples are new. In fact, the justification for writing this paper is to give a unified treatment of why monoids and modules in these categories form model categories. Here we only give an overview; for the details the reader may consult [Se], $[\mathrm{BF}],[\mathrm{Ly}]$ and $[\mathrm{Sch} 2]$ in the case of $\Gamma$-spaces, and [HSS] in the case of symmetric spectra. The particular interest in these categories comes from the fact that they model stable homotopy theory. The homotopy category of symmetric spectra is equivalent to the usual stable homotopy category of algebraic topology. In the case of $\Gamma$-spaces, one obtains the stable homotopy category of connective (i.e., (-1)-connected) spectra. Monoids in either of these categories are thus possible ways of defining 'brave new rings', i.e., rings up to homotopy with higher coherence conditions. Another approach to this idea consists of the $S$-algebras of [EKMM]. 
$\Gamma$-spaces. $\Gamma$-spaces were introduced by G. Segal [Se] who showed that they give rise to a homotopy category equivalent to the usual homotopy category of connective spectra. A. K. Bousfield and E. M. Friedlander $[\mathrm{BF}]$ considered a bigger category of $\Gamma$-spaces in which the ones introduced by Segal appeared as the special $\Gamma$-spaces. Their category admits a simplicial model category structure with a notion of stable weak equivalence giving rise again to the homotopy theory of connective spectra. Then M. Lydakis [Ly] showed that $\Gamma$-spaces admit internal function objects and a symmetric monoidal smash product with nice homotopical properties. Smallness and cofibrant generation for $\Gamma$-spaces is verified in [Sch2], as well as the pushout product and the monoid axiom. The monoids in this setting are called Gamma-rings.

Symmetric spectra. The category of symmetric spectra, $\mathrm{Sp}^{\Sigma}$, is described in [HSS]. There it is also shown that this category is a cofibrantly generated, monoidal model category, and that the associated homotopy category is equivalent to the usual homotopy category of spectra. For symmetric spectra over the category of simplicial sets every object is small with respect to the whole category. The monoid axiom and the fact that smashing with a cofibrant left $R$-module preserves weak equivalences between right $R$-modules are verified in [HSS]. The monoids in this setting are called symmetric ring spectra.

\section{Fibrant examples: simplicial abelian groups, chain complexes and $S$-modules}

These are the examples of monoidal model categories in which every object is fibrant. With this special property it is easier to lift model category structures since the (often hard to verify) condition (1) of the lifting lemma A.3 is a formal consequence of fibrancy and the existence of path objects, see the proof of A.3. For example, the commutative monoids sometimes form model categories in these cases. The pushout product and monoid axioms also hold in these examples, but since the fibrancy property deprives them of their importance, we will not bother to prove them.

Simplicial abelian groups. The model category structure for simplicial abelian groups was established by Quillen [Q, II.6]. The weak equivalences and fibrations are defined on underlying simplicial sets. The cofibrations are the retracts of the free maps (see [Q, II p. 4.11, Rem. 4]). This model category is cofibrantly generated and all objects are small. The (degreewise) tensor product provides a symmetric monoidal product for simplicial abelian groups. The unit for this product is the integers, considered as a constant simplicial abelian group. A monoid then is nothing but a simplicial ring. These have path objects given by the simplicial structure. This means that for a simplicial ring $R$ the simplicial set $\operatorname{Hom}(\Delta[1], R)$ of maps of the standard 1 -simplex into the underlying simplicial set of $R$ is naturally a simplicial ring. The model category structure for simplicial rings and simplicial modules was established by Quillen in $[\mathrm{Q}$, II.4, Thm. 4] and [Q, II.6].

Chain complexes. The category of non-negatively graded chain complexes over a commutative ring $k$ forms a model category, see [Q, II p. 4.11, Remark 5], [DS, Section 7]. The weak equivalences are the maps inducing homology isomorphisms, the fibrations are the maps which are surjective in positive degrees, and cofibrations are monomorphisms with degreewise projective cokernels. This model category is cofibrantly generated and every object is small. The category of unbounded chain complexes over $k$, although less well known, also forms a cofibrantly generated model category with weak equivalences the homology isomorphism and fibrations the epimorphisms, see [HPS], remark after Thm. 9.3.1. The cofibrations here are still degreewise split injections, but their description is a bit more complicated than for bounded chain complexes. The following remarks refer to this category of $\mathbb{Z}$-graded chain complexes of $k$-modules. 
The graded tensor product of chain complexes is symmetric monoidal and has adjoint internal hom-complexes. A monoid in this symmetric monoidal category is a differential graded algebra (DGA). Every complex is fibrant and associative DGAs have path objects. To construct them, we need the following 2-term complex denoted $I$. In degree $0, I$ consists of a free $k$-module on two generators [0] and [1]. In degree $1, I$ is a free $k$-module on a single generator $\iota$. The differential is given by $d \iota=[1]-[0]$. This complex becomes a coassociative and counital coalgebra when given the comultiplication

$$
\Delta: I \longrightarrow I \otimes_{k} I
$$

defined by $\Delta([0])=[0] \otimes[0], \Delta([1])=[1] \otimes[1], \Delta(\iota)=[0] \otimes \iota+\iota \otimes[1]$. The counit map $I \longrightarrow k$ sends both [0] and [1] to $1 \in k$. The two inclusions $k \longrightarrow I$ given by the generators in degree 0 and the counit are maps of coalgebras. Note that the comultiplication of $I$ is not cocommutative (this is reminiscent of the failure of the Alexander-Whitney map to be commutative).

For any coassociative, counital differential graded coalgebra $C$, and any DGA $A$, the internal Hom-chain complex $\operatorname{Hom}_{\mathrm{Ch}}(C, A)_{*}$ becomes a DGA with multiplication

$$
f \cdot g=\mu_{A} \circ(f \otimes g) \circ \Delta_{C}
$$

where $\mu_{A}$ is the multiplication of $A$ and $\Delta_{C}$ is the comultiplication of $C$. In particular, $\operatorname{Hom}_{\mathrm{Ch}}(I, A)$ is a DGA, and it comes with DGA maps from $A$ and to $A \times A$ which make it into a path object. In this way we recover the model category structure for associative DGAs over a commutative ring, first discovered by J. F. Jardine [J]. Our approach is a bit more general, since we can define similar path objects for associative DGAs over a fixed commutative DGA, and for modules over a fixed DGA $A$. We thus also get model categories in those cases. However, since the basic differential graded coalgebra $I$ is not cocommutative, this does not provide path objects for commutative DGAs.

$S$-modules. The model category of $S$-modules, $\mathcal{M}_{S}$, is described in [EKMM, VII 4.6]. This model category structure is cofibrantly generated (see [EKMM, VII 5.6 and 5.8]). To ease notation, let $F_{q}=S \wedge_{\mathcal{L}} \mathbb{L} \Sigma_{q}^{\infty}(-)$, the functor from topological spaces to $\mathcal{M}_{S}$ that is used to define the model category structure on $S$-modules. In our terminology, the generating (acyclic) cofibrations are obtained by applying $F_{q}$ to the generators for topological spaces, $S^{n} \longrightarrow C S^{n}$ $\left(C S^{n} \longrightarrow C S^{n} \wedge I_{+}\right)$, where $C X$ is the cone on $X$. The associative monoids are the $S$-algebras. The difficult part for showing that model category structures can be lifted to the categories of modules and algebras in this case is verifying the smallness hypothesis. This is where the "Cofibration Hypothesis" comes in, see [EKMM, VII 5.2]. The underlying category of S-modules is a topological model category, see [EKMM, VII 4.4] and the triples in question are continuous. Hence, Remark 3.5 applies to give path objects, recovering [EKMM, VII 4.7], in particular the model category structures for $R$-algebras and $R$-modules. Our module comparison theorem 3.3 recovers [EKMM, III 4.2]. Our method of comparing algebra categories over equivalent commutative monoids does not apply here because the unit of the smash product is not cofibrant.

\section{Proofs}

Proof of Theorem 3.1 (1). The category of $R$-modules is also the category of algebras over the triple $T_{R}$ where $T_{R}(M)=R \wedge M$. The triple structure for $T_{R}$ comes from the multiplication $R \wedge R \longrightarrow R$. This theorem is a direct application of Lemma A.3 since by the monoid axiom, the $J_{T}$-cofibrations are weak equivalences. 
Proof of Theorem 3.1 (2). The model category part is Theorem 3.1 (1). By Lemma 2.3, it suffices to check the pushout product axiom and the monoid axiom for the generating (acyclic) cofibrations. Every generating (acyclic) cofibration is induced from $\mathcal{C}$ by smashing with $R$, i.e. it is of the form

$$
R \wedge A \longrightarrow R \wedge B
$$

for $A \longrightarrow B$ a(n) (acyclic) cofibration in $\mathcal{C}$. In the pushout product of two such maps, one $R$ smash factor cancels due to using $\wedge_{R}$, so that the pushout product is again induced from a pushout product of (acyclic) cofibrations in $\mathcal{C}$, where the pushout product axiom holds. This gives the pushout product axiom for $\wedge R$.

If $J$ is a set of generating acyclic cofibrations in $\mathcal{C}$, the set of generating acyclic cofibrations in the category of $R$-modules (called $J_{T}$ above) consists of maps of $J$ smashed with $R$. We thus have the equality $J_{T} \wedge R(R$-mod $)=J \wedge \mathcal{C}$. Since the forgetful functor $R$-mod $\longrightarrow \mathcal{C}$ preserves colimits (it has a right adjoint $[R,-]),\left(J_{T} \wedge(R\right.$-mod) $)$-cof reg $_{\text {reg }}$ is subset of $(J \wedge \mathcal{C})$-cof reg. The monoid axiom for $\mathcal{C}$ thus implies the monoid axiom for $R$-mod.

Proof of Theorem 3.1 (3). This proof is much longer than the previous ones; it occupies the rest of the paper. The main ingredient here is a filtration of a certain pushout in the monoid category. This filtration is also needed to prove the statement about cofibrant monoids. The crucial step only depends on the weak equivalences and cofibrations in the model category structure. Hence we formulate it in a more general context. The hope is that it can also be useful in a situation where one only has something weaker than a model category, without a notion of fibrations. The following definition captures exactly what is needed.

Definition 5.1 An applicable category is a symmetric monoidal category $\mathcal{C}$ equipped with two classes of morphisms called cofibrations and weak equivalences, satisfying the following axioms.

- $\mathcal{C}$ has pushouts and filtered colimits. The monoidal product preserves colimits in each of its variables.

- Any isomorphism is a weak equivalence and a cofibration. Weak equivalences are closed under composition. Cofibrations and acyclic cofibrations are closed under transfinite composition and cobase change.

- The pushout product and monoid axiom are satisfied.

Of course, any monoidal model category which satisfies the monoid axiom is applicable. We are essentially forgetting all references to fibrations since they play no role in the following filtration argument. Note that the notion of regular cofibrations as defined in Definition 2.2 and Appendix A still makes sense in an applicable category. In the following lemma, let $I$ (resp. $J$ ) be the class of those maps between monoids in $\mathcal{C}$ which are obtained from cofibrations (resp. acyclic cofibrations) in $\mathcal{C}$ by application of the free monoid functor, see $(*)$ below.

Lemma 5.2 If $\mathcal{C}$ is an applicable category, any regular $J$-cofibration is a weak equivalence in the underlying category $\mathcal{C}$. If the unit $\mathbb{I}$ of the smash product is cofibrant, then any regular $I$-cofibration whose source is cofibrant in $\mathcal{C}$ is a cofibration in the underlying category $\mathcal{C}$.

Proof of Theorem 3.1 (3), assuming lemma 5.2. By the already established part (2) of Theorem 3.1, the category of $R$-modules is itself a cofibrantly generated, monoidal model category satisfying the monoid axiom. Also if $\mathbb{I}$ is cofibrant in $\mathcal{C}$, then $R$, the unit for $\wedge_{R}$, is 
cofibrant in $R$-mod. So we can assume that the commutative monoid $R$ is actually equal to the unit $\mathbb{I}$ of the smash product, thus simplifying terminology from " $R$-algebras" to "monoids".

To use Lemma A.3 here we need to recognize monoids in $\mathcal{C}$ as the algebras over the free monoid triple $T$. For an object $K$ of $\mathcal{C}$, define $T(K)$ to be

$$
T(K)=\mathbb{I} \amalg K \amalg(K \wedge K) \amalg \ldots \amalg K^{\wedge n} \amalg \ldots
$$

One can think of $T(K)$ as the 'tensor algebra'. Using that $\wedge$ distributes over the coproduct, $T(K)$ has a monoid structure given by concatenation. The functor $T$ is left adjoint to the forgetful functor from monoids to $\mathcal{C}$. Hence $T$ is also a triple on the category $\mathcal{C}$ and the $T$ algebras are precisely the monoids.

Because the monoidal product is closed symmetric, $\wedge$ commutes with colimits. Hence, the underlying functor of $T$ commutes with filtered colimits, as required for Lemma A.3. The condition on the regular cofibrations is taken care of by Lemma 5.2. Let $f: M \longrightarrow N$ be a cofibration of monoids with $M$ cofibrant in $\mathcal{C}$. Every cofibration of monoids is a retract of a regular $I$-cofibration with $I$ as in Lemma 5.2. Hence $f$ is a retract of a regular $I$-cofibration with source cofibrant in $\mathcal{C}$, hence is a cofibration in $\mathcal{C}$. In particular, a cofibrant monoid is a monoid $M$ such that the unit map $\mathbb{I} \longrightarrow M$ is a cofibration of monoids. Since $\mathbb{I}$ is cofibrant, this implies that the unit map is an underlying cofibration. Hence, $M$ is cofibrant in the underlying category $\mathcal{C}$.

Proof of lemma 5.2 The main ingredient is a filtration of a certain kind of pushout in the monoid category. Consider a map $K \longrightarrow L$ in $\mathcal{C}$, a monoid $X$ and a monoid map $T(K) \longrightarrow X$. We want to describe the pushout in the monoid category of the diagram

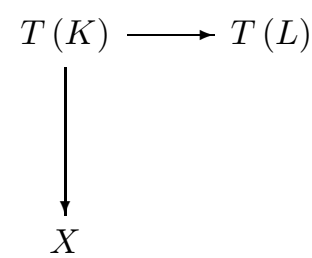

The pushout $P$ will be obtained as the colimit, in the underlying category $\mathcal{C}$, of a sequence

$$
X=P_{0} \longrightarrow P_{1} \longrightarrow \cdots \longrightarrow P_{n} \longrightarrow \cdots .
$$

If one thinks of $P$ as consisting of formal products of elements from $X$ and from $L$, with relations coming from the elements of $K$ and the multiplication in $X$, then $P_{n}$ consists of those products where the total number of factors from $L$ is less than or equal to $n$. For ordinary monoids, this is in fact a valid description, and we will now translate this idea into the element-free form which applies to general symmetric monoidal categories.

As indicated above we set $P_{0}=X$ and describe $P_{n}$ inductively as a pushout in $\mathcal{C}$. We first describe an $n$-dimensional cube in $\mathcal{C}$; by definition, such a cube is a functor

$$
W: \mathcal{P}(\{1,2, \ldots, n\}) \longrightarrow \mathcal{C}
$$

from the poset category of subsets of $\{1,2, \ldots, n\}$ and inclusions to $\mathcal{C}$. If $S \subseteq\{1,2, \ldots, n\}$ is a subset, the vertex of the cube at $S$ is defined to be

$$
W(S)=X \wedge C_{1} \wedge X \wedge C_{2} \wedge \ldots \wedge C_{n} \wedge X
$$


with

$$
C_{i}= \begin{cases}K & \text { if } i \notin S \\ L & \text { if } i \in S\end{cases}
$$

All maps in the cube $W$ are induced from the map $K \longrightarrow L$ and the identity on the $X$ factors.

So at each vertex a total of $n+1$ smash factors of $X$ alternate with $n$ smash factors of either $K$ or $L$. The initial vertex corresponding to the empty subset has all $C_{i}$ 's equal to $K$ and the terminal vertex corresponding to the whole set has all $C_{i}$ 's equal to $L$. For example for $n=2$, the cube is a square and looks like

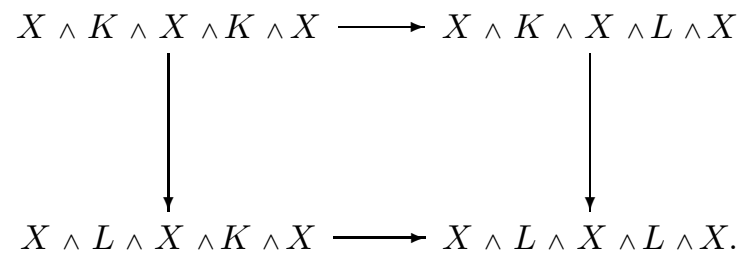

Denote by $Q_{n}$ the colimit of the punctured cube, i.e., the cube with the terminal vertex removed. Define $P_{n}$ via the pushout in $\mathcal{C}$

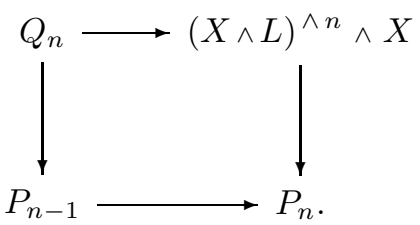

This is not a complete definition until we say what the left vertical map is. We define the map from $Q_{n}$ to $P_{n-1}$ by describing how it maps a vertex $W(S)$ for $S$ a proper subset of $\{1,2, \ldots, n\}$. Each of the smash factors of $W(S)$ which is equal to $K$ is first mapped into $X$. Then adjacent smash factors of $X$ are multiplied. This gives a map

$$
W(S) \longrightarrow X \wedge L \wedge X \wedge \ldots \wedge L \wedge X
$$

where the right hand side has $|S|+1$ smash factors of $X$ and $|S|$ smash factors of $L$. So the right hand side maps further to $P_{|S|}$, hence to $P_{n-1}$ since $S$ is a proper subset.

We have to check that these maps on the vertices of the punctured cube $W$ are compatible so that they assemble to a map from the colimit, $Q_{n}$. So let $S$ be again a proper subset of $\{1,2, \ldots, n\}$ and take $i \notin S$. We have to verify commutativity of the diagram

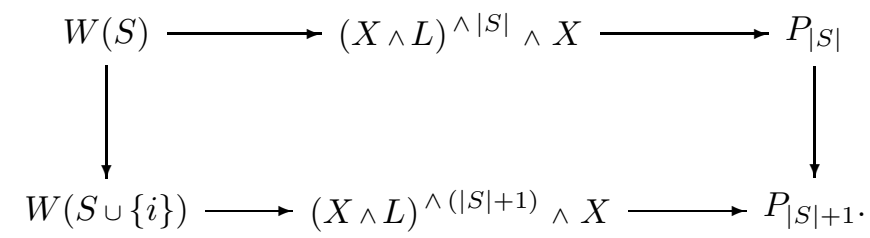


By definition, $W(S)$ and $W(S \cup\{i\})$ differ at exactly one smash factor in the $2 i$-th position which is equal to $K$ for the former and equal to $L$ for the latter. The upper left map factors as

$$
W(S) \longrightarrow(X \wedge L)^{\wedge a} \wedge X \wedge K \wedge(X \wedge L)^{\wedge b} \wedge X \longrightarrow(X \wedge L)^{\wedge|S|} \wedge X
$$

where $a$ (resp. $b$ ) is the number of elements in $S$ which are smaller (resp. larger) than $i$; in particular $a+b=|S|$. The right map in this factorization pushes $K$ into $X$ and multiplies the three adjacent smash factors of $X$. Hence the diagram in question is the composite of two commutative squares

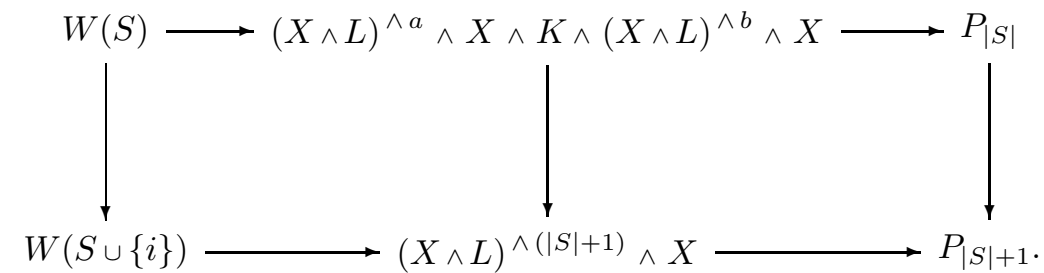

The right square commutes by the definition of $P_{|S|+1}$.

We have now completed the inductive definition of $P_{n}$. We set $P=\operatorname{colim} P_{n}$, the colimit being taken in $\mathcal{C}$. $P$ comes equipped with $\mathcal{C}$-morphisms $X=P_{0} \longrightarrow P$ and

$$
L \cong \mathbb{I} \wedge L \wedge \mathbb{I} \longrightarrow X \wedge L \wedge X \longrightarrow P_{1} \longrightarrow P
$$

which make the diagram

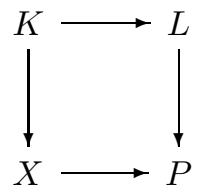

commute. There are several things to check:

(i) $\quad P$ is naturally a monoid so that

(ii) $\quad X \longrightarrow P$ is a map of monoids and

(iii) $P$ has the universal property of the pushout in the category of monoids.

Define the unit of $P$ as the composite of $X \longrightarrow P$ with the unit of $X$. The multiplication of $P$ is defined from compatible maps $P_{n} \wedge P_{m} \longrightarrow P_{n+m}$ by passage to the colimit. These maps are defined by induction on $n+m$ as follows. Note that $P_{n} \wedge P_{m}$ is the pushout in $\mathcal{C}$ in the following diagram.

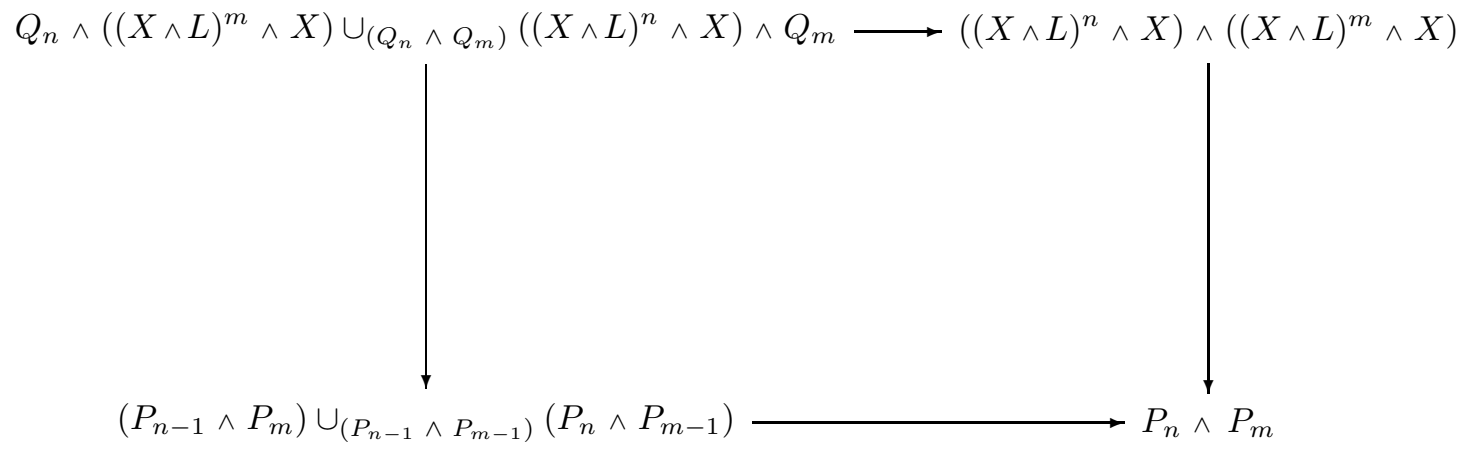


The lower left corner already has a map to $P_{n+m}$ by induction, the upper right corner is mapped there by multiplying the two adjacent factors of $X$ followed by the map $(X \wedge L)^{n+m} \wedge X \longrightarrow$ $P_{n+m}$ from the definition of $P_{n+m}$. We omit the tedious verification that this in fact gives a well defined multiplication map and that the associativity and unital diagrams commute. Hence, $P$ is a monoid. Multiplication in $P$ was arranged so that $X \longrightarrow P$ is a monoid map.

For (iii), suppose we are given another monoid $M$, a monoidal map $X \longrightarrow M$, and a $\mathcal{C}$-map $L \longrightarrow M$ such that the outer square in

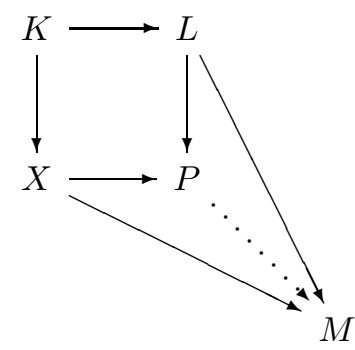

commutes. We have to show that there is a unique monoidal map $P \longrightarrow M$ making the entire square commute. These conditions in fact force the behavior of the composite map $W(S) \longrightarrow P_{n} \longrightarrow P \longrightarrow M$. Since $P$ is obtained by various colimit constructions from these basic building blocks, uniqueness follows. We again omit the tedious verification that the maps $W(S) \longrightarrow M$ are compatible and assemble to a monoidal map $P \longrightarrow M$.

Now that we have established that $P$ is the pushout of the original diagram of monoids, we continue with the homotopical analysis of the constructed filtration, i.e. we will verify that the regular $J$-cofibrations are weak equivalences. Assume now that $K \longrightarrow L$ is an acyclic cofibration in $\mathcal{C}$. The cube $W$ used in the inductive definition of $P_{n}$ has $n+1$ smash factors of $X$ at every vertex which map by the identity everywhere. Using the symmetry isomorphism for $\wedge$, these can all be shuffled to one side and we get that the map $Q_{n} \longrightarrow(X \wedge L)^{\wedge} n \wedge X$ is isomorphic to

$$
\bar{Q}_{n} \wedge X^{\wedge(n+1)} \longrightarrow L^{\wedge n} \wedge X^{\wedge(n+1)} .
$$

Here $\bar{Q}_{n}$ is the colimit of a punctured cube analogous to $W$, but with all the smash factors of $X$ in the vertices deleted. By iterated application of the pushout product axiom, the map $\bar{Q}_{n} \stackrel{\sim}{\longrightarrow} L^{\wedge n}$ is an acyclic cofibration. So by the monoid axiom, the map $P_{n-1} \stackrel{\sim}{\longrightarrow} P_{n}$ is a weak equivalence. The map $X=P_{0} \stackrel{\sim}{\longrightarrow} P$ is an instance of a transfinite composite (indexed by the first infinite ordinal) of the kind of maps considered in the monoid axiom, so it is also a weak equivalence.

With the use of the filtration we just established that any pushout, in the category of monoids, of a map in $J$ is a countable composite of maps of the kind considered in the monoid axiom. A transfinite composite of transfinite composites is again a transfinite composite. Because the forgetful functor from monoids to $\mathcal{C}$ preserves filtered colimits, this shows that regular $J$-cofibrations are weak equivalences.

It remains to prove the statement about regular $I$-cofibrations under the assumption that the unit $\mathbb{I}$ is cofibrant. We note that if in the above pushout diagram $K \longrightarrow L$ is a cofibration and the monoid $X$ is cofibrant in the underlying category, then

$$
\bar{Q}_{n} \wedge X^{\wedge(n+1)} \longrightarrow L^{\wedge n} \wedge X^{\wedge(n+1)}
$$


is a cofibration in the underlying category (by several applications of the pushout product axiom). Thus also the maps $P_{n-1} \longrightarrow P_{n}$ and finally $X=P_{0} \longrightarrow P$ are cofibrations in the underlying category. Since the forgetful functor commutes with filtered colimits, transfinite composites of such pushouts in the monoid category are still cofibrations in the underlying category $\mathcal{C}$.

\section{A Cofibrantly generated model categories}

We need to transfer model category structures to categories of algebras over triples. In [Q, p. II 3.4], Quillen formulates his small object argument, which is now the standard device for such purposes. After Quillen, several authors have axiomatized and generalized the small object argument (see e.g. [Bl, Def. 4.4], [Cr, Def. 3.2] or [Sch1, Def. 1.3.1]). In our context we will need a transfinite version of the small object argument. An axiomatization suitable for our purposes is the 'cofibrantly generated model category' of [DHK], which we now recall.

If a model category is cofibrantly generated, its model category structure is completely determined by a set of cofibrations and a set of acyclic cofibrations. The transfinite version of Quillen's small object argument allows functorial factorization of maps as cofibrations followed by acyclic fibrations and as acyclic cofibrations followed by fibrations. Most of the model categories in the literature are cofibrantly generated, e.g. topological spaces and simplicial sets, as are all the examples that appear in this paper.

Ordinals and cardinals. An ordinal $\gamma$ is an ordered isomorphism class of well ordered sets; it can be identified with the well ordered set of all preceding ordinals. For an ordinal $\gamma$, the same symbol will denote the associated poset category. The latter has an initial object $\emptyset$, the empty ordinal. An ordinal $\kappa$ is a cardinal if its cardinality is larger than that of any preceding ordinal. A cardinal $\kappa$ is called regular if for every set of sets $\left\{X_{j}\right\}_{j \in J}$ indexed by a set $J$ of cardinality less than $\kappa$ such that the cardinality of each $X_{j}$ is less than that of $\kappa$, then the cardinality of the union $\bigcup_{J} X_{j}$ is also less than that of $\kappa$. The successor cardinal (the smallest cardinal of larger cardinality) of every cardinal is regular.

Transfinite composition. Let $\mathcal{C}$ be a cocomplete category and $\gamma$ a well ordered set which we identify with its poset category. A functor $V: \gamma \longrightarrow \mathcal{C}$ is called a $\gamma$-sequence if for every limit ordinal $\beta<\gamma$ the natural map colim $\left.V\right|_{\beta} \longrightarrow V(\beta)$ is an isomorphism. The map $V(\emptyset) \longrightarrow$ $\operatorname{colim}_{\gamma} V$ is called the transfinite composition of the maps of $V$. A subcategory $\mathcal{C}_{1} \subset \mathcal{C}$ is said to be closed under transfinite composition if for every ordinal $\gamma$ and every $\gamma$-sequence $V: \gamma \longrightarrow \mathcal{C}$ with the map $V(\alpha) \longrightarrow V(\alpha+1)$ in $\mathcal{C}_{1}$ for every ordinal $\alpha<\gamma$, the induced map $V(\emptyset) \longrightarrow \operatorname{colim}_{\gamma} V$ is also in $\mathcal{C}_{1}$. Examples of such subcategories are the cofibrations or the acyclic cofibrations in a closed model category.

Relatively small objects. Consider a cocomplete category $\mathcal{C}$ and a subcategory $\mathcal{C}_{1} \subset \mathcal{C}$ closed under transfinite composition. If $\kappa$ is a regular cardinal, an object $C \in \mathcal{C}$ is called $\kappa$-small relative to $\mathcal{C}_{1}$ if for every regular cardinal $\lambda \geq \kappa$ and every functor $V: \lambda \longrightarrow \mathcal{C}_{1}$ which is a $\lambda$-sequence in $\mathcal{C}$, the map

$$
\operatorname{colim}_{\lambda} \operatorname{Hom}_{\mathcal{C}}(C, V) \longrightarrow \operatorname{Hom}_{\mathcal{C}}\left(C, \operatorname{colim}_{\lambda} V\right)
$$

is an isomorphism. An object $C \in \mathcal{C}$ is called small relative to $\mathcal{C}_{1}$ if there exists a regular cardinal $\kappa$ such that $C$ is $\kappa$-small relative to $\mathcal{C}_{1}$. 
$I$-injectives, I-cofibrations and regular $I$-cofibrations. Given a cocomplete category $\mathcal{C}$ and a class $I$ of maps, we denote

- by $I$-inj the class of maps which have the right lifting property with respect to the maps in $I$. Maps in $I$-inj are referred to as $I$-injectives.

- by $I$-cof the class of maps which have the left lifting property with respect to the maps in $I$-inj. Maps in $I$-cof are referred to as I-cofibrations.

- by $I$-cof reg $\subset I$-cof the class of the (possibly transfinite) compositions of pushouts of maps

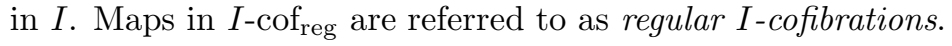

Quillen's small object argument [Q, p. II 3.4] has the following transfinite analogue. Note that here $I$ has to be a set, not just a class of maps.

Lemma A.1 [DHK] Let $\mathcal{C}$ be a cocomplete category and I a set of maps in $\mathcal{C}$ whose domains are small relative to $I$-cof $f_{\text {reg. }}$. Then

- there is a functorial factorization of any map $f$ in $\mathcal{C}$ as $f=q i$ with $q \in I$-inj and $i \in I$ cof $_{\text {reg }}$ and thus

- every I-cofibration is a retract of a regular I-cofibration.

Definition A.2 [DHK] A model category $\mathcal{C}$ is called cofibrantly generated if it is complete and cocomplete and there exists a set of cofibrations $I$ and a set of acyclic cofibrations $J$ such that

- the fibrations are precisely the $J$-injectives;

- the acyclic fibrations are precisely the $I$-injectives;

- the domain of each map in $I$ (resp. in $J$ ) is small relative to $I$-cof ${ }_{\text {reg }}\left(\right.$ resp. $J$-cof ${ }_{\text {reg }}$ ).

Moreover, here the (acyclic) cofibrations are the $I(J)$-cofibrations.

For a specific choice of $I$ and $J$ as in the definition of a cofibrantly generated model category, the maps in $I$ (resp. $J$ ) will be referred to as generating cofibrations (resp. generating acyclic cofibrations). In cofibrantly generated model categories, a map may be functorially factored as an acyclic cofibration followed by a fibration and as a cofibration followed by an acyclic fibration.

Let $\mathcal{C}$ be a cofibrantly generated model category and $T$ a triple on $\mathcal{C}$. We want to form a model category on the category of algebras over the triple $T$, denoted $T$-alg. Call a map of $T$-algebras a weak equivalence (resp. fibration) if the underlying map in $\mathcal{C}$ is a weak equivalence (resp. fibration). Call a map of $T$-algebras a cofibration if it has the left lifting property with respect to all acyclic fibrations. The forgetful functor $T$-alg $\longrightarrow \mathcal{C}$ has a left adjoint, the free functor $F^{T}$. The following lemma gives two different situations in which one can lift a model category on $\mathcal{C}$ to one on $T$-alg. We make no great claim to originality for this lemma. Other lifting theorems for model category structures can be found in [Bl, Thm. 4.14], [CG, Thm. 2.5], [Cr, Thm. 3.3], [DHK, II 8.2], [EKMM, VII Thm. 4.7, 4.9]. 
Let $X$ be a $T$-algebra. We define a path object for $X$ to be a $T$-algebra $X^{I}$ together with $T$-algebra maps

$$
X \stackrel{\sim}{\longrightarrow} X^{I} \longrightarrow X \times X
$$

factoring the diagonal map, such that the first map is a weak equivalence and the second map is a fibration in the underlying category $\mathcal{C}$.

Lemma A.3 Assume that the underlying functor of $T$ commutes with filtered direct limits. Let $I(J)$ be a set of generating cofibrations (resp. acyclic cofibrations) for the cofibrantly generated model category $\mathcal{C}$. Let $I_{T}$ (resp. $J_{T}$ ) be the image of these sets under the free T-algebra functor. Assume that the domains of $I_{T}\left(J_{T}\right)$ are small relative to $I_{T}$-cof $f_{\text {reg }}\left(J_{T}\right.$-cof $\left.f_{\text {reg }}\right)$. Suppose

1. every regular $J_{T}$-cofibration is a weak equivalence, or

2. every object of $\mathcal{C}$ is fibrant and every $T$-algebra has a path object.

Then the category of T-algebras is a cofibrantly generated model category with $I_{T}\left(J_{T}\right)$ the generating set of (acyclic) cofibrations.

Proof: We refer the reader to [DS, 3.3] for the numbering of the model category axioms. All those kinds of limits that exist in $\mathcal{C}$ also exist in $T$-alg, and limits are created in the underlying category $\mathcal{C}$ [Bor, Prop. 4.3.1]. Colimits are more subtle, but since the underlying functor of $T$ commutes with filtered colimits, they exist by [Bor, Prop. 4.3.6]. Model category axioms MC2 (saturation) and MC3 (closure properties under retracts) are clear. One half of MC4 (lifting properties) holds by definition of cofibrations of $T$-algebras.

The proof of the remaining axioms uses the transfinite small object argument, which exists here because of Lemma A.1, and the hypothesis about the smallness of the domains.

We begin with the factorization axiom, MC5. Every map in $I_{T}$ and $J_{T}$ is a cofibration of $T$-algebras by adjointness. Hence any $I_{T}$-cofibration or $J_{T}$-cofibration is a cofibration of $T$ algebras. By adjointness and the fact that $I$ is a generating set of cofibrations for $\mathcal{C}$, a map is $I_{T}$-injective precisely when the map is an acyclic fibration of underlying objects, i.e., an acyclic fibration of $T$-algebras. Hence the small object argument applied to the set $I_{T}$ gives a (functorial) factorization of any map in $T$-alg as a cofibration followed by an acyclic fibration.

The other half of the factorization axiom, MC5, needs hypothesis (1) or (2). Applying the small object argument to the set of maps $J_{T}$ gives a functorial factorization of a map in $T$ alg as a regular $J_{T}$-cofibration followed by a $J_{T}$-injective. Since $J$ is a generating set for the acyclic cofibrations in $\mathcal{C}$, the $J_{T}$-injectives are precisely the fibrations among the $T$-algebra maps, once more by adjointness. In case (1) we assume that every regular $J_{T}$-cofibration is a weak equivalence on underlying objects in $\mathcal{C}$. We noted above that every $J_{T}$-cofibration is a cofibration in $T$-alg. So we see that the factorization above is an acyclic cofibration followed by a fibration.

In case (2) we can adapt the argument of [Q, II p.4.9] as follows. Let $i: X \longrightarrow Y$ be any $J_{T}$-cofibration. We claim that it is a weak equivalence in the underlying category. Since $X$ is fibrant and fibrations are $J_{T}$-injectives, we obtain a retraction $r$ to $i$ by lifting in the square

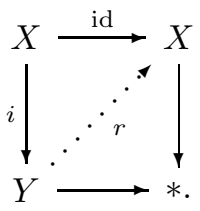


$Y$ possesses a path object and $i$ has the LLP with respect to fibrations. So a lifting exists in the square

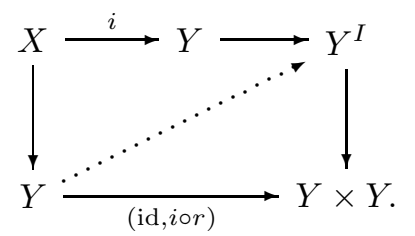

This shows that in the homotopy category of $\mathcal{C}, i \circ r$ is equal to the identity map of $Y$. Since maps in $\mathcal{C}$ are weak equivalences if and only if they become isomorphisms in the homotopy category of $\mathcal{C}$, this proves that $i$ is a weak equivalence, and it finishes the proof of model category axiom MC5 under hypothesis (2).

It remains to prove the other half of MC4, i.e., that any acyclic cofibration $A \stackrel{\sim}{\longrightarrow} B$ has the LLP with respect to fibrations. In other words, we need to show that the acyclic cofibrations are contained in the $J_{T}$-cofibrations. The small object argument provides a factorization

$$
A \stackrel{\sim}{\longrightarrow} W \longrightarrow B
$$

with $A \longrightarrow W$ a $J_{T}$-cofibration and $W \longrightarrow B$ a fibration. In addition, $W \longrightarrow B$ is a weak equivalence since $A \longrightarrow B$ is. Since $A \longrightarrow B$ is a cofibration, a lifting in

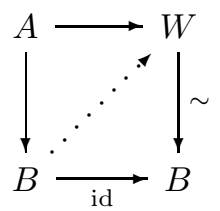

exists. Thus $A \longrightarrow B$ is a retract of a $J_{T}$-cofibration, hence a $J_{T}$-cofibration.

Remark A.4 Hypothesis (2) can be weakened to the existence of a fibrant replacement functor in the category of $T$-algebras which interacts well with respect to the path object, see [Sch2, Lemma A.3]. Quillen's argument in [Q, II p.4.9] in fact uses Kan's Ex ${ }^{\infty}$ functor as such a fibrant replacement functor.

Remark A.5 To simplify the exposition, we assume that every object of $\mathcal{C}$ is small relative to the whole category $\mathcal{C}$ when we apply lemma A.3 in the rest of this paper. This holds for $\Gamma$-spaces and symmetric spectra based on simplicial sets. If the underlying functor of the triple $T$ on $\mathcal{C}$ commutes with filtered direct limits, then so does the forgetful functor from $T$-algebras to $\mathcal{C}$. Hence by adjointness, every free $T$-algebra is small relative to the whole category of $T$-algebras, so the smallness conditions of lemma A.3 hold. Of course, if one is interested in a category where not all objects are small with respect to all of $\mathcal{C}$ one must verify those smallness conditions directly. 


\section{References}

[Bl] D. Blanc: New model categories from old, J. Pure Appl. Algebra 109 (1996), 37-60

[Bor] F. Borceux: Handbook of Categorical Algebra 2: Categories and Structures, Encyclopedia of Mathematics and its Applications 51, Cambridge University Press (1994)

[BF $] \quad$ A. K. Bousfield, E. M. Friedlander: Homotopy theory of $\Gamma$-spaces, spectra, and bisimplicial sets, Springer Lecture Notes 658 (1978), 80-130

[CG] J. Cabello, A. Garzón: Closed model structures for algebraic models of $n$-types, J. Pure Appl. Algebra 103, 287-302

[Cr] S. E. Crans: Quillen closed model category structures for sheaves, J. Pure Appl. Algebra 101 (1995), 35-57

[DHK $] \quad$ W. G. Dwyer, P. S. Hirschhorn, D. M. Kan: Model categories and more general abstract homotopy theory, in preparation

[DS] W. G. Dwyer, J. Spalinski: Homotopy theories and model categories, in: Handbook of algebraic topology, ed. I. M. James, Elsevier (1995)

[EKMM] A. D. Elmendorf, I. Kriz, M. A. Mandell, J. P. May, with an appendix by M. Cole: Rings, Modules, and Algebras in Stable Homotopy Theory, Mathematical Surveys and Monographs 47, AMS (1997)

[GZ] P. Gabriel, M. Zisman: Calculus of fractions and homotopy theory, Ergebnisse der Mathematik und ihrer Grenzgebiete 35 (1967), Springer

[HPS] M. Hovey, J. Palmieri, N. Strickland: Axiomatic stable homotopy theory, Mem. Amer. Math. Soc. 128 (1997), no. 610.

[HSS] M. Hovey, B. Shipley, J. Smith: Symmetric spectra, preprint

[J] J. F. Jardine: A closed model category structure for differential graded algebras, preprint

[Lw] L. G. Lewis, Jr.: Is there a convenient category of spectra? J. Pure Appl. Algebra 73 (1991), 233-246

[Ly] M. Lydakis: Smash-products and $\Gamma$-spaces, to appear, Math. Proc. Cam. Phil. Soc.

[MacL] S. Mac Lane: Categories for the working mathematician, Springer (1971)

[Q] D. Quillen: Homotopical Algebra, Springer Lecture Notes 43 (1967)

[Sch1] S. Schwede: Spectra in model categories and applications to the algebraic cotangent complex, to appear, J. Pure Appl. Algebra

[Sch2] S. Schwede: Stable homotopical algebra and $\Gamma$-spaces, to appear, Math. Proc. Cam. Phil. Soc.

[Se] G. Segal: Categories and cohomology theories, Topology 13 (1974), 293-312

Fakultät für Mathematik

Universität Bielefeld

33615 Bielefeld, Germany

schwede@mathematik.uni-bielefeld.de
Department of Mathematics

University of Chicago

Chicago, IL 60637, USA

bshipley@math.uchicago.edu 